\title{
Relationship between milk cathelicidin abundance and microbiologic culture in clinical mastitis
}

\author{
M. F. Addis, ${ }^{\star 1}$ V. Bronzo, $\dagger$ G. M. G. Puggioni, ${ }^{*}$ C. Cacciotto, ${ }^{*}$ V. Tedde, ${ }^{\star}$ D. Pagnozzi, ${ }^{\star}$ C. Locatelli, $\ddagger$ A. Casula, $\dagger$ \\ G. Curone, $\dagger$ S. Uzzau, ${ }^{*} \S^{2}$ and P. Moroni†\# ${ }^{2}$ \\ *Porto Conte Ricerche, SP 55 Porto Conte/Capo Caccia, Km 8.400, Loc. Tramariglio, 07041 Alghero, Italy \\ †Dipartimento di Medicina Veterinaria, Università degli Studi di Milano, Via Celoria 10, 20133 Milan, Italy \\ fDipartimento di Scienze Veterinarie per la Salute, la Produzione Animale e la Sicurezza Alimentare, Università degli Studi di Milano, \\ Via Celoria 10, 20133 Milan, Italy \\ §Dipartimento di Scienze Biomediche, Università degli Studi di Sassari, Viale S. Pietro 43/B, 07100 Sassari, Italy \\ \#Quality Milk Production Services, Animal Health Diagnostic Center, Cornell University, 240 Farrier Road, Ithaca, NY 14853
}

\begin{abstract}
The availability of reliable tools to enable the sensitive and specific detection of mastitis in dairy cows can assist in developing control strategies and promote the more rational use of antibiotics. We have developed a milk cathelicidin ELISA that shows high sensitivity and specificity for dairy cow mastitis, based on latent class analysis. In this study, we investigated the effect of microbial agents on cathelicidin abundance in the milk of cows with clinical mastitis. We subjected 535 quarter milk samples (435 from quarters showing signs of clinical mastitis and 100 from healthy quarters as a control) to milk cathelicidin ELISA, somatic cell count (SCC), and microbiologic culture. Of the 435 clinical mastitis samples, $431(99.08 \%)$ were positive for cathelicidin, $424(97.47 \%)$ had SCC >200,000 cells/ $\mathrm{mL}$, and $376(86.44 \%)$ were culture-positive. Of the 59 culture-negative samples, $58(98.30 \%)$ were positive for cathelicidin and $55(93.22 \%)$ had SCC >200,000 cells/ $\mathrm{mL}$. The abundance of cathelicidin and the extent of SCC increase depended on the causative agent: Streptococcus agalactiae and coagulase-negative staphylococci showed the highest and lowest changes, respectively. We also observed differences in behavior between the 2 markers depending on the pathogen: Streptococcus agalactiae induced the highest cathelicidin abundance, and Serratia spp. induced the highest SCC. Nevertheless, the different ability of microorganisms to induce cathelicidin release in milk did not compromise its value as a mastitis marker, given its higher sensitivity compared to SCC or microbiologic culture. All 100 negative control samples (collected from healthy quarters with
\end{abstract}

Received October 5, 2016.

Accepted November 24, 2016.

${ }^{1}$ Corresponding author: addis@portocontericerche.it

${ }^{2}$ These authors contributed equally.
SCC $<100,000$ cells/mL and culture-negative) were also negative for cathelicidin, corresponding to $100 \%$ specificity in the evaluated sample cohort. This study confirmed the value of the milk cathelicidin ELISA for detecting bovine mastitis, and highlighted the influence of mastitis-causing microorganisms on cathelicidin abundance. This influence did not compromise diagnostic performance; instead, it may have better reflected disease severity and evolution than SCC.

Key words: clinical mastitis, cathelicidin, dairy cow, ELISA, intramammary infection

\section{INTRODUCTION}

Sensitive and specific tools for the detection of mastitis in dairy cows are needed to reduce its economic impact. Effective diagnostic methods can lead to faster and more efficient control of mastitis in dairy ruminants and promote the more responsible use of antibiotic therapy. At present, mastitis monitoring is based mainly on SCC, but the immunologic detection of inflammation markers may be a valuable alternative, providing practical benefits and enabling the improvement of diagnostic performance (Viguier et al., 2009; Ceciliani et al., 2012). One of the most promising inflammation markers is cathelicidin, a class of proteins with antimicrobial activity and potent proinflammatory and chemotactic functions (Zanetti, 2004, 2005). Cathelicidin is released in milk, first by epithelial cells upon contact with an invading pathogen, and then by activated PMNL retrieved into milk by degranulation and the formation of neutrophil extracellular traps (Addis et al., 2013; Pisanu et al., 2015).

We have recently developed a high-performing milk pan-cathelicidin ELISA that shows elevated sensitivity and specificity for dairy cow mastitis relative to SCC and microbial culture (Addis et al., 2016b). We estimated the assay's performance using latent class 
analysis in a Bayesian framework (that is, without referring to a gold standard for true disease status). Other authors have observed the release of cathelicidin in milk in natural and experimental infections (Smolenski et al., 2011; Wheeler et al., 2012; Reinhardt et al., 2013). However, in their pioneering study, Smolenski et al. (2011) reported a lack of cathelicidin reactivity in about $25 \%$ of mastitis milk samples using immunoblotting analysis. They hypothesized, among other causes, that different microbial infections could induce different levels of cathelicidin release, compromising its diagnostic sensitivity, but the analytical technique and antibodies they used may also have influenced the sensitivity and specificity of pan-cathelicidin detection.

We identified the need to assess the influence of microbial agents on cathelicidin abundance as measured by our pan-cathelicidin ELISA, and to evaluate their effect on the diagnostic value of the ELISA compared to SCC. We investigated the relationships among cathelicidin, SCC, and culture results by considering the presence of clearly detectable clinical signs (clinical mastitis) as the gold standard for true disease status.

\section{MATERIALS AND METHODS}

\section{Milk Sample Collection, Microbiologic Culture, and SCC}

Milk samples were obtained from 16 dairy herds between March and May 2016. The farms were required to have trained technicians able to detect clinical mastitis; use an adequate milking routine that included forestripping to detect abnormal milk; and sample any case of clinical mastitis and freeze quarter milk immediately. Clinical mastitis was defined as the presence of udder or quarter swelling; heat, hardness, redness, or pain; or visible alterations in the milk, such as watery appearance, flakes, clots, or pus. Sample collection was carried out after careful cleaning and disinfection of teat ends with chlorhexidine-embedded disposable towels. For each sample, approximately $10 \mathrm{~mL}$ of milk was collected into a sterile vial after discharging the first stream. Samples were stored at $-20^{\circ} \mathrm{C}$ and sent to the laboratory weekly for microbiological analysis and SCC determination. A total of 535 quarter milk samples were included in the sample cohort: 435 from cows with clinical mastitis, and 100 from clinically healthy cows with negative microbiologic culture and SCC $<100,000$ cells $/ \mathrm{mL}$.

At the laboratory of the Department of Veterinary Medicine, University of Milan, milk samples were thawed at room temperature, and $100 \mu \mathrm{L}$ was spread onto blood agar plates ( $5 \%$ defibrinated sheep blood). Plates were incubated aerobically at $37^{\circ} \mathrm{C}$ and evaluated after 24 and $48 \mathrm{~h}$. Microbiologic culture was carried out following guidelines from the National Mastitis Council (1999), with the exception of a $10 \times$ increase in inoculum volume. Provisional identification of colonies was based on Gram stain, morphology, and hemolysis patterns. Representative colonies were subcultured on blood agar plates and incubated again at $37^{\circ} \mathrm{C}$ for $24 \mathrm{~h}$. Gram-positive, catalase-positive cocci were evaluated using the coagulase tube test to differentiate Staphylococcus aureus from other staphylococci. Gram-positive, catalase-negative cocci were identified as streptococci and differentiated by further biochemical tests (growth in $6.5 \% \mathrm{NaCl}$ broth, esculin hydrolysis, fermentation of ribose, sorbitol, sucrose, and inulin) and by the Christie, Atkins, and Munch-Petersen test. Gram-negative bacteria were identified using Gram stain characteristics, oxidase reaction, and colony morphology on MacConkey's agar (Oxoid, Basingstoke, UK) and Eosin Methylene Blue agar (Laboratorios Conda, Madrid, Spain). Microorganisms other than bacteria were confirmed by microscopic appearance. When the growth of 2 different microorganisms was detected ( 25 samples of $376,6.65 \%$ ), the case was classified as a mixed infection. Samples with growth of 3 or more pathogens were considered contaminated and were not included in the study. We determined SCC using an automated somatic cell counter (Bentley Somacount 150; Bentley Instruments, Chaska, MN).

\section{Pan-Cathelicidin ELISA}

Cathelicidin abundance in milk was assessed at the Porto Conte Ricerche laboratories with a pan-cathelicidin sandwich ELISA based on 2 monoclonal antibodies developed against a pan-cathelicidin domain, as previously described (Addis et al., 2016a,b). At the end of the assay, the optical density at $450 \mathrm{~nm}$ (OD450) value of all samples was normalized against internal controls. To this aim, 6 culture-negative samples with $<50,000$ cells $/ \mathrm{mL}$ were included in all ELISA plates, and their average OD450 (+3 SD) was subtracted from all OD450 values to obtain the normalized OD450 value (NOD450). To assess cathelicidin abundance, each milk sample was tested in duplicate aliquots of 10 and $1 \mu \mathrm{L}$. When the results of the $10-\mu \mathrm{L}$ aliquot provided a NOD450 higher than 2.5, we considered the NOD of the $1-\mu \mathrm{L}$ aliquot multiplied by 10 . Finally, to enable comparison and logarithmic visualization, we added a correction factor of 0.1 to all NOD450 values to obtain the adjusted OD450 value (AOD450). 


\section{Statistics}

The Shapiro-Wilk normality test indicated that the data followed a non-normal distribution. Therefore, statistical significance of the differences among result distributions was evaluated using the Kruskal-Wallis test with the Dunn's post-test correction. Statistical analysis and descriptive statistics [median and interquartile range (IQR)] were carried out using GraphPad Prism version 5.03 for Windows (GraphPad Software, La Jolla, CA). To assess diagnostic performance, we applied thresholds of 0.115 AOD450 for the cathelicidin ELISA (Addis et al., 2016b), and 200,000 cells/mL for SCC (Dohoo and Leslie, 1991; Schukken et al., 2003; Bradley and Green, 2005). To evaluate sensitivity and specificity, we considered the presence of clinical signs to be the gold standard for true disease status.

\section{RESULTS}

\section{Cathelicidin ELISA, SCC, and Culture Results}

We evaluated the 535 quarter milk samples by cathelicidin ELISA, SCC, and culture, and assessed diagnostic performance based on the presence of clinical mastitis as true disease status. The results obtained from the 435 clinical mastitis samples are summarized in Table 1: 431 of 435 were positive for cathelicidin (sensitivity 99.08\%), 424 of 435 had SCC > 200,000 cells/mL (sensitivity $97.47 \%$ ), and 376 of 435 were culture-positive (sensitivity 86.44\%). Streptococcus uberis, Escherichia coli, Streptococcus agalactiae, and CNS were the most commonly isolated microorganisms. Of the 59 culture-negative clinical mastitis samples, 58 (98.30\%) were positive for cathelicidin, 55 (93.22\%) had SCC > 200,000 cells $/ \mathrm{mL}$, and 1 was negative for both markers. All 100 milk samples from healthy mammary quarters (culture-negative and SCC $<100,000$ cells $/ \mathrm{mL}$ ) were negative for cathelicidin, indicating a specificity of $100 \%$.

\section{Cathelicidin Abundance and SCC in Clinical and Healthy Mammary Quarters}

To estimate cathelicidin abundance in milk, we tested aliquots of 1 and $10 \mu \mathrm{L}$ by ELISA to calculate AOD450. Figure 1 illustrates the resulting distribution for cathelicidin and SCC in all samples, classified as healthy; all clinical mastitis; culture-positive clinical mastitis; and culture-negative clinical mastitis. Healthy quarters were significantly different from clinical mastitis quarters $(P$ $\leq 0.001$ ), both as a group and when considered separately for culture status. The median AOD450 value was slightly higher in culture-positive than in culture- negative clinical mastitis, but this difference was not statistically significant. All clinical mastitis samples but 4 for cathelicidin (3 culture-positive and 1 culturenegative) and 11 for SCC (7 culture-positive and 4 culture-negative) were above positivity thresholds. The median and IQR for cathelicidin AOD450 and SCC in all samples are reported in Table 2 .

\section{Cathelicidin Abundance and SCC According to the Pathogen Group}

We analyzed the distribution of cathelicidin and SCC results from clinical mastitis milk samples based on the relevance of the isolated microorganisms as IMI agents (Harmon, 1994). Specifically, we considered the following groups to compare cathelicidin and SCC results: contagious pathogens (Streptococcus agalactiae and Staphylococcus aureus), environmental pathogens (Streptococcus spp. and gram-negative), CNS, other microorganisms, mixed infections, and culture-negative samples (Figure 2). According to the Kruskal-Wallis test, result distributions were significantly different for both cathelicidin and SCC, with a more pronounced effect for the former. In the pairwise comparisons, cathelicidin abundance in IMI by Streptococcus agalactiae was significantly different from all other groups, except for Staphylococcus aureus and mixed infections. Cathelicidin abundance in IMI by CNS was also significantly

Table 1. Summary of the results obtained from the 435 clinical mastitis samples

\begin{tabular}{lcc}
\hline Item & $\begin{array}{c}\text { No. of } \\
\text { samples }\end{array}$ & $\%$ \\
\hline Cathelicidin ELISA & $431 / 435$ & 99.08 \\
SCC > 200,000 cells/mL & $424 / 435$ & 97.47 \\
Microbiologic culture & $376 / 435$ & 86.44 \\
Streptococcus uberis & 66 & 15.17 \\
Escherichia coli & 63 & 14.48 \\
Streptococcus agalactiae & 59 & 13.56 \\
CNS & 41 & 9.43 \\
Streptococcus dysgalactiae & 26 & 5.98 \\
Mixed infection & 25 & 5.75 \\
Klebsiella spp. & 17 & 3.91 \\
Staphylococcus aureus & 14 & 3.22 \\
Enterococcus faecalis & 10 & 2.30 \\
Lactococcus lactis & 9 & 2.07 \\
Serratia spp. & 7 & 1.61 \\
Corynebacterium spp. & 7 & 1.61 \\
Yeast & 6 & 1.38 \\
Streptococcus bovis & 5 & 1.15 \\
Other gram-negative & 5 & 1.15 \\
Enterobacter spp. & 3 & 0.69 \\
Pasteurella spp. & 3 & 0.69 \\
Aerococcus viridans & 3 & 0.69 \\
Streptococcus spp. & 2 & 0.46 \\
Pseudomonas spp. & 2 & 0.46 \\
Citrobacter spp. & 2 & 0.46 \\
Trueperella pyogenes & 1 & 0.23 \\
\hline & & \\
\hline
\end{tabular}


A

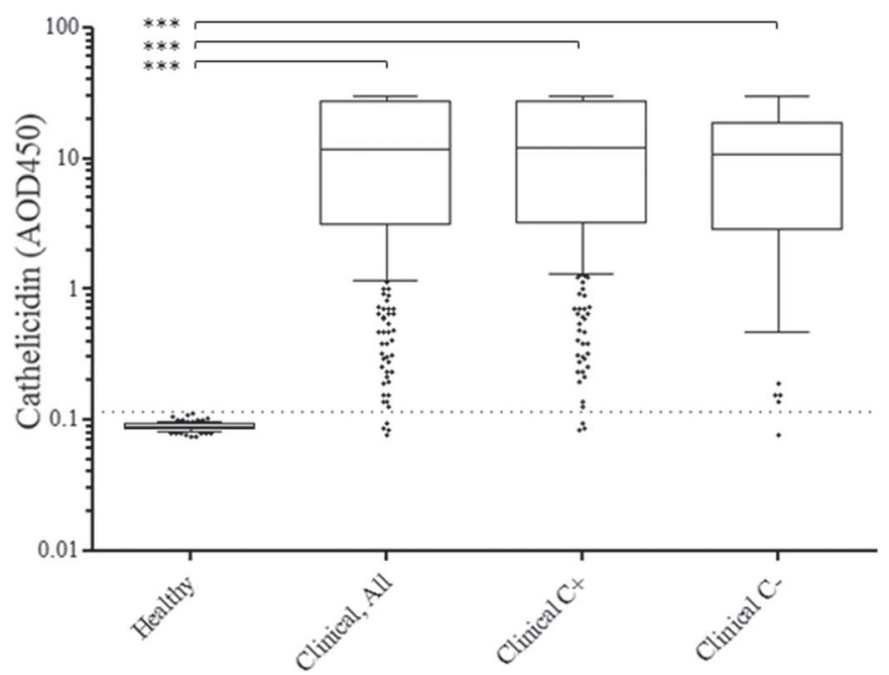

$\mathrm{B}$

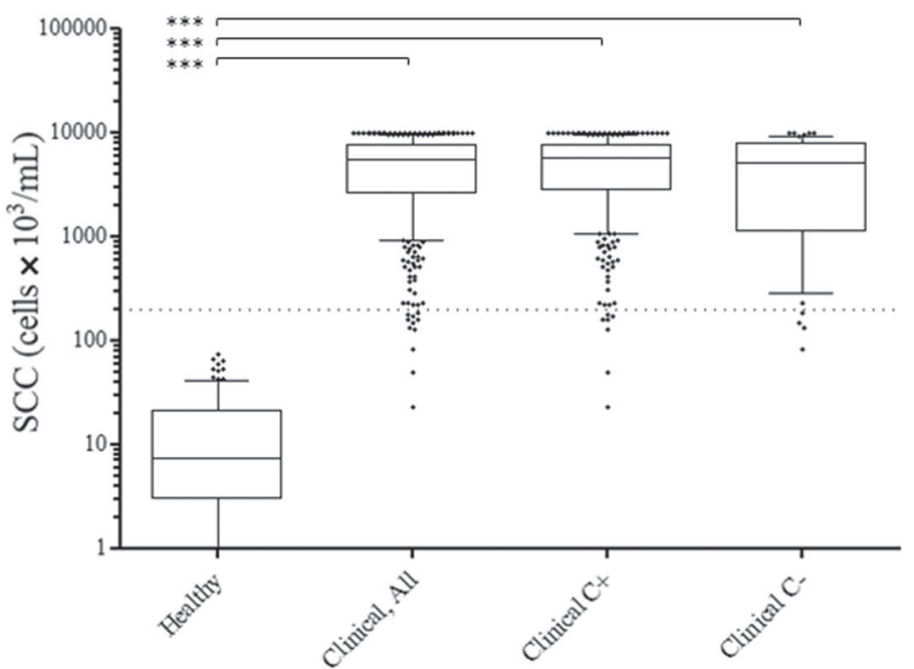

Figure 1. Distribution on a $\log _{10}$ scale of (A) cathelicidin and (B) SCC values measured in the 535 samples, according to clinical condition and culture $(\mathrm{C})$ results. Boxes indicate values within the 25 th and 75 th percentiles, and the central line indicates the median value. Whiskers indicate values within the 10th and 90th percentiles, and individual dots represent values outside the whiskers. Dashed lines indicate the positivity threshold for each plot: adjusted optical density at $450 \mathrm{~nm}$ (AOD450) of 0.115 for cathelicidin and $200 \times 10^{3}$ cells $/ \mathrm{mL}$ for SCC. Statistically significant differences (***P $\leq 0.001$ ) among classes, according to a Kruskal-Wallis test with Dunn's post-test correction, are indicated by a continuous line.

different for Streptococcus spp. and gram-negative groups (Figure 2A). In the case of SCC (Figure 2B), we observed a statistically significant difference only in IMI by Streptococcus agalactiae compared with IMI by CNS. Statistically significant differences and their respective $P$-values are outlined in Figure $2 \mathrm{C}$ for cathelicidin and Figure 2D for SCC. Table 3 reports descriptive statistics for the different microorganism classes, listed in decreasing order of median abundance for cathelicidin and SCC, respectively.

\section{Cathelicidin Abundance and SCC According to the Isolated Microorganism}

To obtain further detail on differences in microorganisms' ability to induce cathelicidin release, we also analyzed cathelicidin abundance and SCC by considering separately each IMI agent identified in a minimum of 6 clinical milk samples (Figure 3). Microorganisms that did not satisfy these criteria were grouped as "other gram-positive" or "other gram-negative."

We observed statistically significant differences for cathelicidin abundance in IMI by Streptococcus agalactiae compared with 6 of 15 microorganism groups, and in IMI by CNS compared with 3 of 15 microorganism groups (Figures 3A and 3C). We did not observe any statistically significant differences among groups for SCC (Figure 3B and 3D).

Table 4 reports the median and IQR for cathelicidin (AOD450) and SCC (cells/mL) according to decreasing order of abundance in IMI by different microorganisms. We observed several differences for the 2 parameters. The microorganism inducing the highest cathelicidin abundance (median AOD450 of 27.480) was Streptococ-

Table 2. Median and interquartile range (IQR) of cathelicidin and SCC

\begin{tabular}{llrr}
\hline Item & Sample class & Median & IQR \\
\hline Cathelicidin $\left(\mathrm{AOD} 450^{1}\right)$ & Healthy & 0.089 & $0.084-0.094$ \\
& Clinical mastitis, all & 11.850 & $3.090-27.120$ \\
& Clinical mastitis, culture-positive & 12.260 & $3.170-27.495$ \\
SCC $($ cells $/ \mathrm{mL})$ & Clinical mastitis, culture-negative & 70.620 & $2.803-18.920$ \\
& Healthy & 5,500 & $3,000-21,000$ \\
& Clinical, all & $5,692,000$ & $2,540,000-7,814,000$ \\
& Clinical mastitis, culture-positive & $5,049,000$ & $2,778,000-7,752,000$ \\
& Clinical mastitis, culture-negative & $1,106,000-7,992,000$ & \\
\hline
\end{tabular}

\footnotetext{
${ }^{1}$ Adjusted optical density at $450 \mathrm{~nm}$.
} 
cus agalactiae; Serratia spp. induced the lowest cathelicidin abundance (median AOD450 of 2.474). Enterococcus faecalis induced the highest SCC increase (median SCC of $8,184,000$ cells $/ \mathrm{mL}$ ), followed by Serratia spp. (median SCC of $7,330,000$ ), in contrast to cathelicidin. Streptococcus agalactiae ranked only fourth (median
SCC of $6,362,000$ cells $/ \mathrm{mL}$ ), and CNS induced the lowest SCC increase (median SCC of 2,808,000 cells/mL).

We observed no statistically significant correlation of microbial load with cathelicidin abundance or SCC increase, collectively or by microorganism or microorganism group.
A

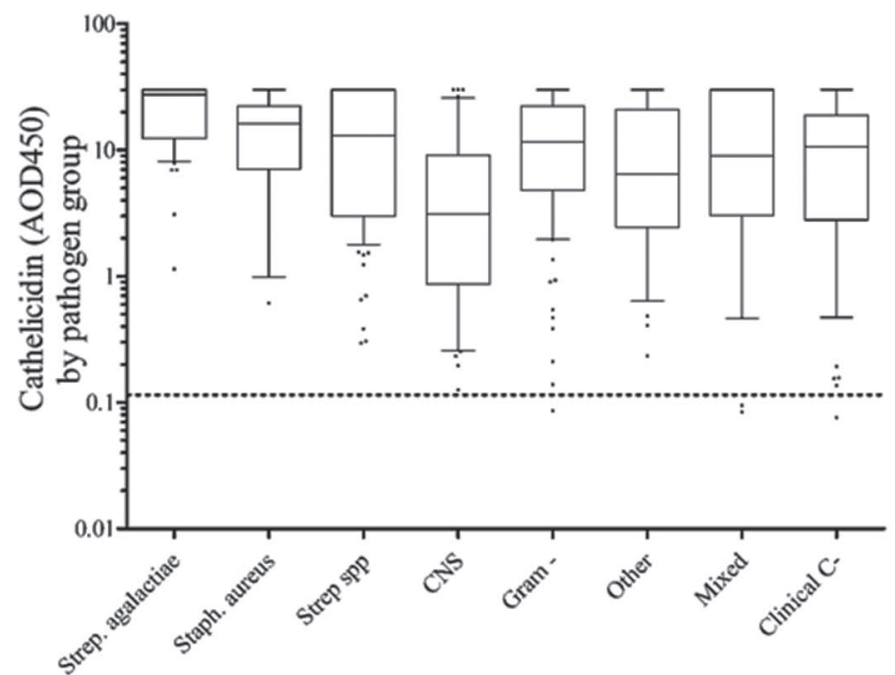

C

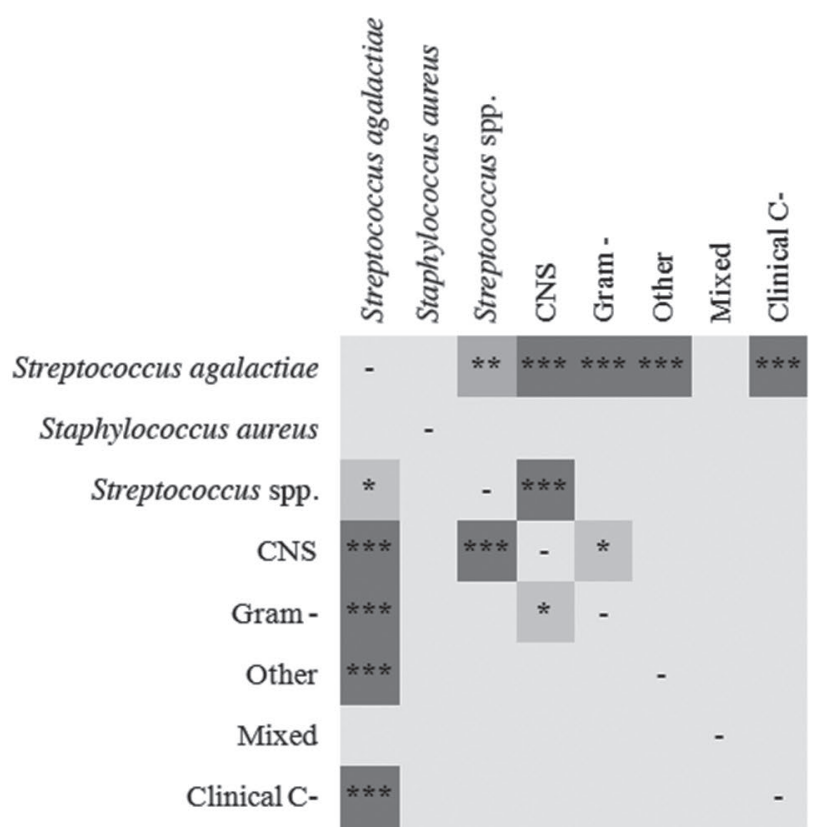

B

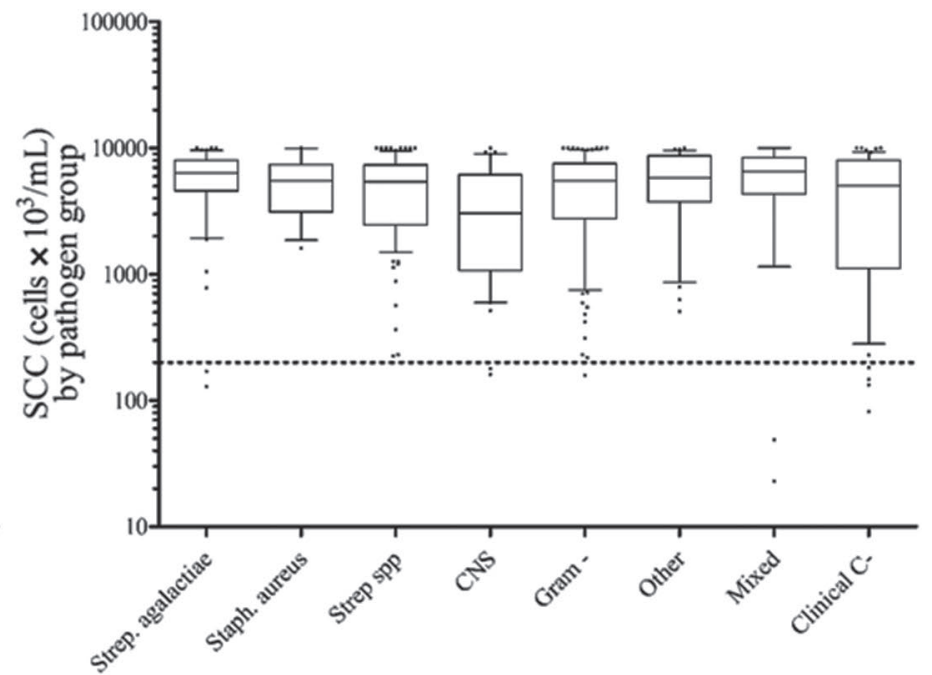

$\mathrm{D}$

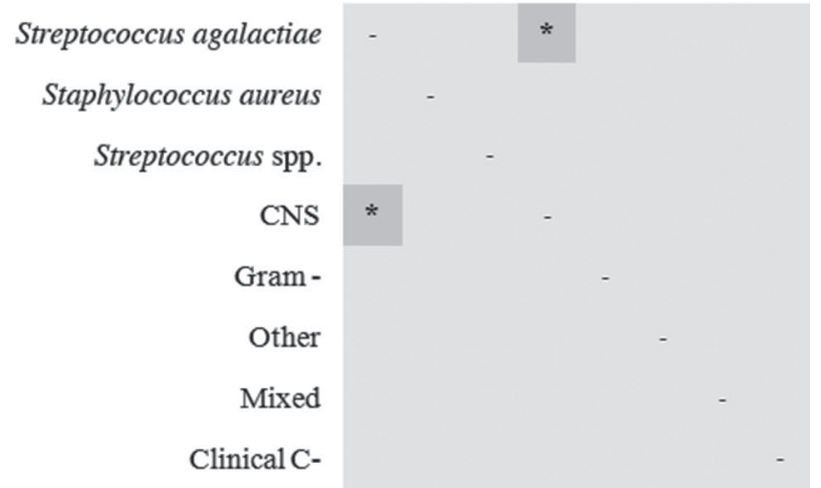

Figure 2. Top: Distribution on a $\log _{10}$ scale of (A) cathelicidin, and (B) SCC values measured in the 435 samples, according to pathogen group. Boxes indicate values within the 25 th and 75 th percentiles, and the central line indicates the median value. Whiskers indicate values within the 10th and 90th percentiles, and individual dots represent values outside the whiskers. Dashed lines indicate the positivity threshold for each plot: adjusted optical density at $450 \mathrm{~nm}$ (AOD450) of 0.115 for cathelicidin and $200 \times 10^{3}$ cells $/ \mathrm{mL}$ for SCC. Bottom: Matrix tables summarizing the statistical significance of the differences for (C) cathelicidin, and (D) SCC. ${ }^{* * *} P \leq 0.001,{ }^{* *} P \leq 0.01,{ }^{*} P \leq 0.05$. C- $=$ culture-negative. 


\section{DISCUSSION}

Cathelicidins are a class of potent antimicrobial and proinflammatory proteins involved in innate immune response to infection. These proteins are released quickly and abundantly following an IMI, and therefore have the potential to be early and sensitive markers of mastitis (Addis et al., 2013; Smolenski et al., 2014). We recently developed a pan-cathelicidin ELISA with higher sensitivity than SCC and comparable specificity (Addis et al., 2016a,b). Previous studies have suggested that different microorganisms might induce different levels of cathelicidin release in milk (Smolenski et al., 2011). To assess the extent of these differences and their possible effect on diagnostic performance, we investigated cathelicidin abundance and SCC in mammary quarters with clinical mastitis resulting from different microorganisms.

Almost all clinical samples were positive for cathelicidin, showing a sensitivity higher than SCC $>200,000$ cells $/ \mathrm{mL}$ (99.08 vs. $97.47 \%$, respectively). In a previous article, we reported sensitivities of $80.6,74.4$, and $38.8 \%$, and specificities of $94.9,96.3$, and $92.8 \%$, for the cathelicidin ELISA, SCC >200,000 cells $/ \mathrm{mL}$, and microbiologic culture, respectively (Addis et al., 2016b). In that report, we evaluated diagnostic performance in a population that included healthy, clinical mastitis, and subclinical mastitis quarters, and we implemented a latent class analysis approach because of the lack of a gold standard for true disease status (Hui and Walter, 1980; Koop et al., 2011; van Smeden et al., 2014). Accordingly, we noted that cathelicidin specificity might be underestimated by using the latent class analysis approach, given its higher sensitivity (Addis et al., 2016a). In the present study, by selecting true positives (clinical mastitis samples) and reliable negatives (healthy, very low SCC, culture-negative samples) as the gold standards for true disease and healthy status, we confirmed the superior sensitivity of the cathelicidin ELISA and highlighted its elevated specificity.

Culture-negative mastitis remains an issue. In fact, 10 to $40 \%$ of clinical mastitis cases yield a negative culture result, and this number seems to be increasing (Makovec and Ruegg, 2003). This issue is due to numerous and complex factors, including fastidiousness or the inability of some microorganisms to grow in culture; the presence of microbes below detection thresholds; antibiotic treatment; or the presence of host-produced antimicrobial molecules, mastitis caused by non-bacterial microorganisms, or mastitis due to dysbiosis (Oikonomou et al., 2014). In the present study, we observed a slightly lower median for culture-negative clinical mastitis samples than for culture-positive samples, but the difference was not statistically significant. Therefore, culture negativity should not be expected to affect the diagnostic performance of cathelicidin.

When we examined the results of culture-positive clinical mastitis samples according to pathogen group or microorganism, we found that the abundance of milk cathelicidin was significantly influenced by the mastitis agent, but cathelicidin levels always remained well above the diagnostic threshold. In this way, we confirmed the observations of Smolenski et al. (2011) on the differential release of cathelicidin in milk according to pathogen. However, these authors reported a lack of reactivity in about $25 \%$ of culture-positive clinical mastitis samples, and we observed a sensitivity of close to $100 \%$. This difference might be due to poorer detection performance of the antibodies or the technique Smolenski et al. (2011) employed for detection (Western immunoblotting). We observed a slightly lower sensitivity with Western immunoblotting compared with ELISA with the same anti-pan-cathelicidin monoclonal antibodies used for this study (data not shown).

The microorganisms that displayed the most pronounced differences in cathelicidin abundance were

Table 3. Median and interquartile range (IQR) of cathelicidin and SCC observed for the different microorganism groups ${ }^{1}$

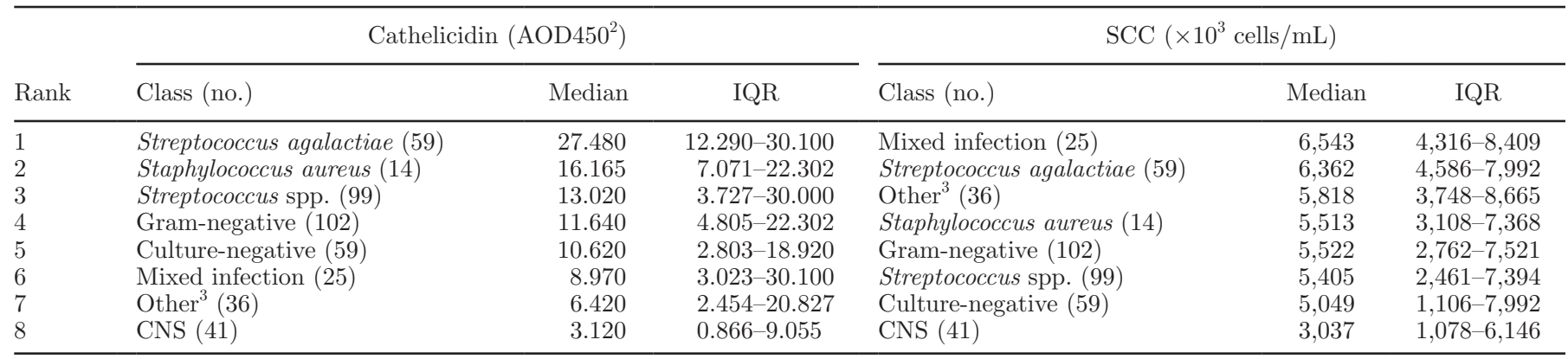

${ }^{1}$ Groups are reported in decreasing order (rank) according to respective median value.

${ }^{2} \mathrm{AOD} 450$ = adjusted optical density at $450 \mathrm{~nm}$.

${ }^{3}$ Other: Enterococcus faecalis, Lactococcus lactis, Corynebacterium spp., yeast, Aerococcus viridans, Trueperella pyogenes. 

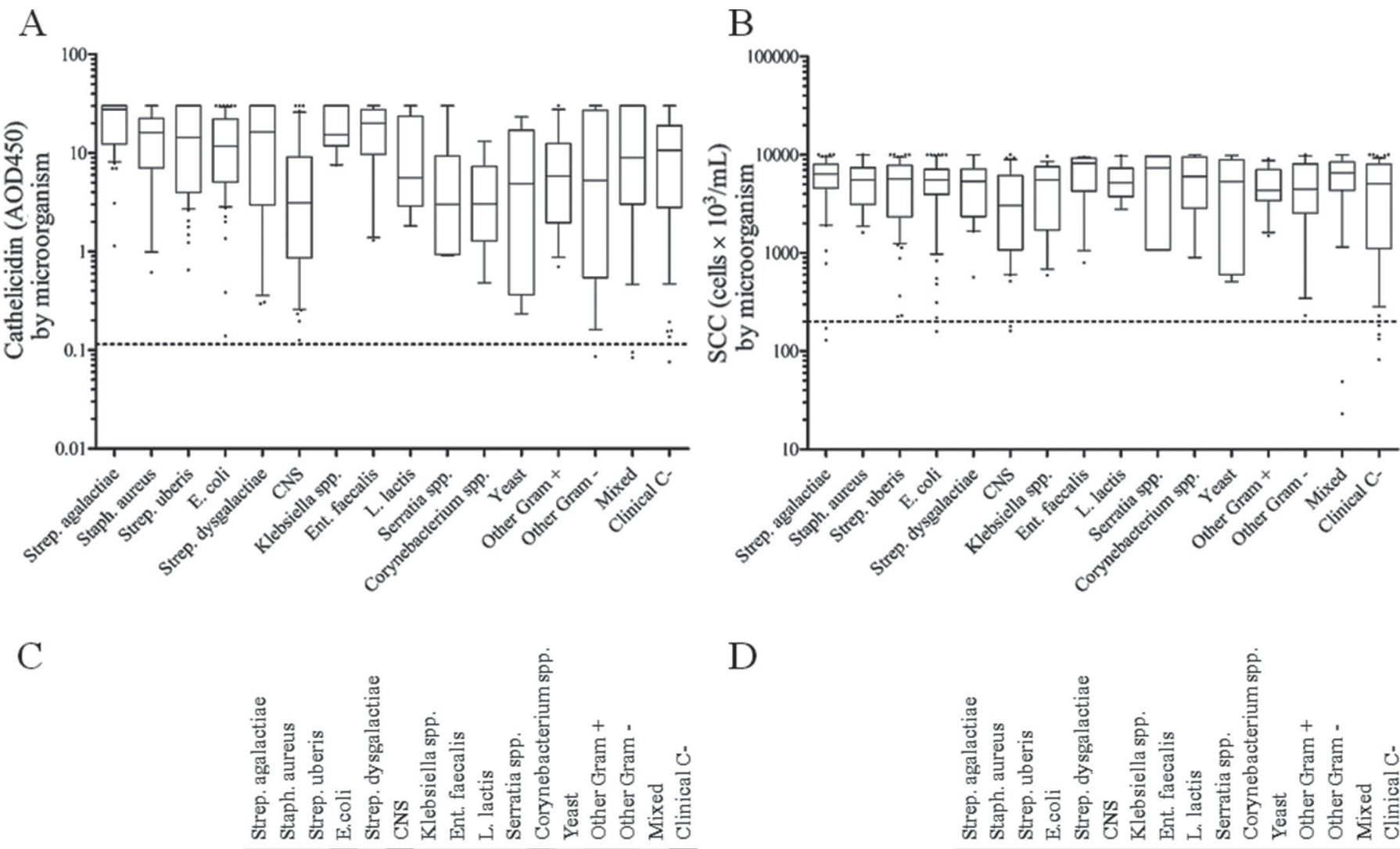

$\mathrm{D}$
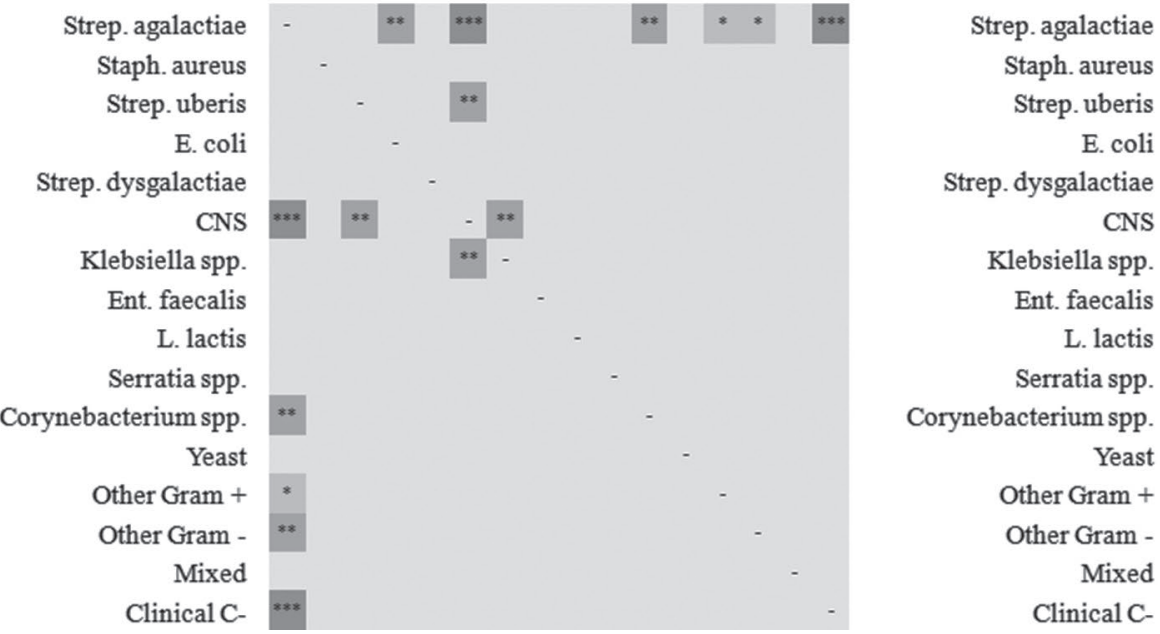

Figure 3. Top: Distribution on a $\log _{10}$ scale of (A) cathelicidin, and (B) SCC values measured in 435 samples, according to microbiologic culture results. Boxes indicate values within the 25 th and 75 th percentiles, and the central line indicates the median value. Whiskers indicate values within the 10th and 90th percentiles, and individual dots represent values outside the whiskers. Dashed lines indicate the positivity threshold for each plot: adjusted optical density at $450 \mathrm{~nm}$ (AOD450) of 0.115 for cathelicidin and $200 \times 10^{3}$ cells $/ \mathrm{mL}$ for SCC. Bottom: Matrix tables summarizing the statistical significance of the differences for (C) cathelicidin, and (D) SCC. ${ }^{* * *} P \leq 0.001, * * P \leq 0.01, * P \leq 0.05$. C- $=$ culture-negative.

Streptococcus agalactiae and CNS, which produced the highest and lowest AOD450 values, respectively. In the case of cathelicidin, Streptococcus agalactiae and CNS also differed significantly from almost all other pathogen groups, in agreement with their respective roles as mastitis pathogens. Streptococcus agalactiae is a major contagious mastitis agent, together with Staphylococcus aureus; accordingly, the latter induced the second-highest increase in cathelicidin abundance, and its differences compared with the former were not statistically 
significant. On the other hand, CNS were among the lowest inducers, in agreement with their lesser potential as acute udder pathogens (Schukken et al., 2009).

Taking the microorganisms separately, we observed several differences, although the methods used to make isolate identifications at the genus and species level may have included some misclassification error, particularly for the streptococcal organisms, because phenotypic methods can be inadequate. One striking observation was the lower ability of Serratia spp. and Corynebacterium spp. to induce cathelicidin increase, in contrast to SCC increase. The reason for this has yet to be investigated, but this finding might be due to these microorganisms' different ability to recall PMNL compared to other cells in milk. On the other hand, the factors leading to higher abundance of cathelicidin in milk can be numerous and complex, and may include more efficient PMNL recruitment, PMNL degranulation, or neutrophil extracellular trap formation ( $\mathrm{Lu}$ et al., 2012), as well as the ability of the pathogen to mediate PMNL lysis (Oliver et al., 1998; Kobayashi et al., 2010; Le Maréchal et al., 2013). Adding to these factors, microbial resistance to lysis by antimicrobial peptides, with mechanisms including repulsion, digestion, sequestration, or excretion (Yeaman and Yount, 2003; Kraus and Peschel, 2006; Joo and Otto, 2015), may also contribute to observed differences in cathelicidin abundance. According to Wheeler et al. (2012), milk cathelicidin-derived antimicrobial peptides display different growth-suppression activities for different mastitis-causing microorganisms. Those authors re- ported a $\mathrm{MIC}_{50}$ of $8 \mu \mathrm{g} / \mathrm{mL}$ for a cathelicidin extract against different gram-negative pathogens (Escherichia coli, Pseudomonas aeruginosa, Salmonella typhimurium) compared with a $\mathrm{MIC}_{50}$ of $33 \mu \mathrm{g} / \mathrm{mL}$ against Streptococcus uberis. Chaneton et al. (2008) observed that the ability of Streptococcus uberis to resist the antimicrobial agent lactoferrin was probably responsible for the very high abundance of this protein in the milk of animals with Streptococcus uberis mastitis. Microbial species that are associated with higher concentrations of antimicrobial molecules may be less susceptible to their activity than more sensitive microorganisms. That is, the mammary gland may respond to microbial infection by increasing the secretion of antimicrobial proteins in a pathogen-specific manner, and pathogenic agents that trigger a greater response may have adapted to these conditions by becoming increasingly resistant to their antimicrobial activity. In addition, when considering that phagocytosis and other PMNL defense mechanisms are inhibited by milk (Lippolis et al., 2006), the role of antimicrobial peptides in the biology of mastitis becomes even more relevant (Pisanu et al., 2015; Cacciotto et al., 2016).

The advantages of a sensitive and specific mastitis test based on protein markers linked to innate immunity rather than somatic cells are numerous (Viguier et al., 2009) and have been discussed in depth in our recent articles describing the cathelicidin ELISA (Addis et al., 2016a,b). Among these are the ability to use immunoassays suitable for the laboratory, the field, and in-line systems, as well as the closer correlation

Table 4. Median and interquartile range (IQR) of cathelicidin and SCC observed for the different microorganisms in the 435 clinical samples ${ }^{1}$

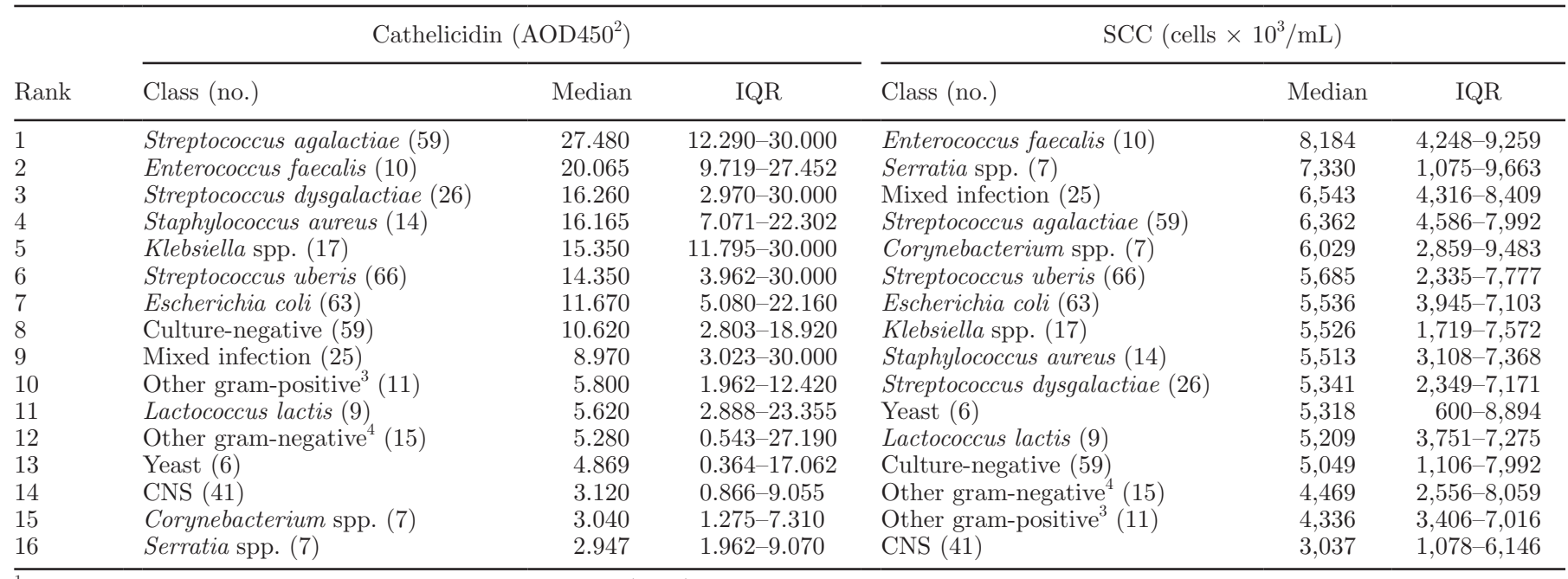

${ }^{1}$ Microorganism classes are reported in decreasing order (rank) according to respective median value.

${ }^{2} \mathrm{AOD} 450$ = adjusted optical density at $450 \mathrm{~nm}$.

${ }^{3}$ Other gram-positives: Aerococcus viridans, Streptococcus bovis, Streptococcus spp., Trueperella pyogenes.

${ }^{4}$ Other gram-negatives: gram-negative bacilli, Enterobacter spp., Pasteurella spp., Pseudomonas spp., Citrobacter spp. 
of protein markers with the presence of inflammation. Because of the elevated sensitivity and specificity demonstrated in the present study, cathelicidin may find application in improving the detection of subclinical and clinical mastitis in automated milking systems and for helping identify mammary quarters eligible for selective dry cow therapy.

Given that diagnostic performance was maintained, the correlation of cathelicidin abundance with mastitis pathogens may provide several advantages. In fact, cathelicidin may reflect different aspects of disease biology and evolution compared with SCC, being more closely related to the number of activated PMNL inside the mammary gland. As such, it may enable to monitor the success of therapy more efficiently (Kawai et al., 2015) and it may find utility as a research tool in mastitis, such as for investigating the ability of a pathogen to resist lysis by antimicrobial peptides or to induce inflammation and PMNL recruitment, activation, and killing. On the other hand, the influence of the mastitis pathogen on cathelicidin levels does not possess enough discriminatory power to guide treatment decisions, and culture will still be needed to select therapeutic interventions.

\section{CONCLUSIONS}

Abundance of cathelicidin in milk demonstrates its potential as mastitis marker, and we confirmed the high sensitivity and specificity of a cathelicidin ELISA in this study. Although different pathogens induced cathelicidin release to different extents, the diagnostic value of this marker was not compromised. Instead, such differences may be a better reflection of microbial pathogenicity; they may more closely represent disease severity and be useful for disease classification, investigating the ability of different pathogens to cause mastitis, monitoring disease recovery, providing indications on the success of mastitis treatment, or guiding selection of mammary quarters eligible for selective dry cow treatments.

\section{ACKNOWLEDGMENTS}

This work was funded by Regione Autonoma della Sardegna with funds granted to the Sardinia Regional Science and Technology Park.

\section{REFERENCES}

Addis, M. F., S. Pisanu, G. Marogna, T. Cubeddu, D. Pagnozzi, C. Cacciotto, F. Campesi, G. Schianchi, S. Rocca, and S. Uzzau. 2013. Production and release of antimicrobial and immune defense proteins by mammary epithelial cells following Streptococcus uberis infection of sheep. Infect. Immun. 81:3182-3197. https:// doi.org/10.1128/IAI.00291-13.

Addis, M. F., V. Tedde, S. Dore, S. Pisanu, G. M. G. Puggioni, A. M. Roggio, D. Pagnozzi, and S. Lollai. 2016a. Evaluation of milk cathelicidin for detection of dairy sheep mastitis. J. Dairy Sci. 99:6446-6456. https://doi.org/10.3168/jds.2015-10293.

Addis, M. F., V. Tedde, G. M. G. Puggioni, S. Pisanu, A. Casula, C. Locatelli, N. Rota, V. Bronzo, P. Moroni, and S. Uzzau. 2016b. Evaluation of milk cathelicidin for detection of bovine mastitis. J. Dairy Sci. 99:8250-8258. https://doi.org/10.3168/jds.2016-11407.

Bradley, A., and M. Green. 2005. Use and interpretation of somatic cell count data in dairy cows. In Pract. 27:310-315. https://doi. org/10.1136/inpract.27.6.310.

Cacciotto, C., T. Cubeddu, M. F. Addis, A. G. Anfossi, V. Tedde, G. Tore, T. Carta, S. Rocca, B. Chessa, M. Pittau, and A. Alberti 2016. Mycoplasma lipoproteins are major determinants of neutrophil extracellular trap formation. Cell. Microbiol. 18:1751-1762. https://doi.org/10.1111/cmi.12613.

Ceciliani, F., J. J. Ceron, P. D. Eckersall, and H. Sauerwein. 2012. Acute phase proteins in ruminants. J. Proteomics 75:4207-4231. https://doi.org/10.1016/j.jprot.2012.04.004.

Chaneton, L., L. Tirante, J. Maito, J. Chaves, and L. E. Bussmann. 2008. Relationship between milk lactoferrin and etiological agent in the mastitic bovine mammary gland. J. Dairy Sci. 91:18651873. https://doi.org/10.3168/jds.2007-0732.

Dohoo, I. R., and K. E. Leslie. 1991. Evaluation of changes in somatic cell counts as indicators of new intramammary infections. Prev. Vet. Med. 10:225-237. https://doi.org/10.1016/0167-5877(91)90006-N.

Harmon, R. J. 1994. Physiology of mastitis and factors affecting somatic cell counts. J. Dairy Sci. 77:2103-2112. https://doi.org/10.3168/ jds.S0022-0302(94)77153-8.

Hui, S. L., and S. D. Walter. 1980. Estimating the error rates of diagnostic tests. Biometrics 36:167-171.

Joo, H. S., and M. Otto. 2015. Mechanisms of resistance to antimicrobial peptides in staphylococci. Biochim. Biophys. Acta 1848:30553061. https://doi.org/10.1016/j.bbamem.2015.02.009.

Kawai, K., K. Korematsu, K. Akiyama, M. Okita, Y. Yoshimura, and N. Isobe. 2015. Dynamics of lingual antimicrobial peptide, lactoferrin concentrations and lactoperoxidase activity in the milk of cows treated for clinical mastitis. Anim. Sci. J. 86:153-158. https://doi.org/10.1111/asj.12269.

Kobayashi, S. D., K. R. Braughton, A. M. Palazzolo-Ballance, A. D Kennedy, E. Sampaio, E. Kristosturyan, A. R. Whitney, D. E. Sturdevant, D. W. Dorward, S. M. Holland, B. N. Kreiswirth, J. M. Musser, and F. R. DeLeo. 2010. Rapid neutrophil destruction following phagocytosis of Staphylococcus aureus. J. Innate Immun. 2:560-575. https://doi.org/10.1159/000317134.

Koop, G., T. van Werven, N. Toft, and M. Nielen. 2011. Estimating test characteristics of somatic cell count to detect Staphylococcus aureus-infected dairy goats using latent class analysis. J. Dairy Sci. 94:2902-2911. https://doi.org/10.3168/jds.2010-3929.

Kraus, D., and A. Peschel. 2006. Molecular mechanisms of bacteria resistance to antimicrobial peptides. Curr. Top. Microbiol. Immunol. 306:231-250.

Le Maréchal, C., J. Jardin, V. Briard-Bion, L. Rault, N. Berkova, E. Vautor, R. Thiéry, S. Even, and Y. Le Loir. 2013. Staphylococcus aureus proteins differentially produced in ewe gangrenous mastitis or ewe milk. Vet. Microbiol. 164:150-157. https://doi. org $/ 10.1016 /$ j.vetmic.2013.01.013.

Lippolis, J. D., T. A. Reinhardt, J. P. Goff, and R. L. Horst. 2006 Neutrophil extracellular trap formation by bovine neutrophils is not inhibited by milk. Vet. Immunol. Immunopathol. 113:248-255. https://doi.org/10.1016/j.vetimm.2006.05.004.

Lu, T., S. D. Kobayashi, M. T. Quinn, and F. R. Deleo. 2012. A NET outcome. Front. Immunol. 3:365 https://doi.org/10.3389/ fimmu.2012.00365.

Makovec, J. A., and P. L. Ruegg. 2003. Results of milk samples submitted for microbiological examination in Wisconsin from 1994 to 2001. J. Dairy Sci. 86:3466-3472. https://doi.org/10.3168/jds. S0022-0302(03)73951-4. 
National Mastitis Council. 1999. Laboratory Handbook on Bovine Mastitis. National Mastitis Council, Madison, WI.

Oikonomou, G., M. L. Bicalho, E. Meira, R. E. Rossi, C. Foditsch, V. S. Machado, A. G. V. Teixeira, C. Santisteban, Y. H. Schukken, and R. C. Bicalho. 2014. Microbiota of cow's milk; distinguishing healthy, sub-clinically and clinically diseased quarters. PLoS One 9:e85904 https://doi.org/10.1371/journal.pone.0085904.

Oliver, S. P., R. A. Almeida, and L. F. Calvinho. 1998. Virulence factors of Streptococcus uberis isolated from cows with mastitis. Zentralbl, Veterinärmed. B 45:461-471.

Pisanu, S., T. Cubeddu, D. Pagnozzi, S. Rocca, C. Cacciotto, A. Alberti, G. Marogna, S. Uzzau, and M. F. Addis. 2015. Neutrophil extracellular traps in sheep mastitis. Vet. Res. (Faisalabad) 46:59. https://doi.org/10.1186/s13567-015-0196-x.

Reinhardt, T. A., R. E. Sacco, B. J. Nonnecke, and J. D. Lippolis. 2013. Bovine milk proteome: Quantitative changes in normal milk exosomes, milk fat globule membranes and whey proteomes resulting from Staphylococcus aureus mastitis. J. Proteomics 82:141-154. https://doi.org/10.1016/j.jprot.2013.02.013.

Schukken, Y. H., R. N. González, L. L. Tikofsky, H. F. Schulte, C. G. Santisteban, F. L. Welcome, G. J. Bennett, M. J. Zurakowski, and R. N. Zadoks. 2009. CNS mastitis: Nothing to worry about? Vet. Microbiol. 134:9-14.

Schukken, Y. H., D. J. Wilson, F. Welcome, L. Garrison-Tikofsky, and R. N. Gonzalez. 2003. Monitoring udder health and milk quality using somatic cell counts. Vet. Res. 34:579-596. https://doi. org/10.1051/vetres:2003028.

Smolenski, G. A., M. K. Broadhurst, K. Stelwagen, B. J. Haigh, and T. T. Wheeler. 2014. Host defence related responses in bovine milk during an experimentally induced Streptococcus uberis infection. Proteome Sci. 12:19. https://doi.org/10.1186/1477-5956-12-19.

Smolenski, G. A., R. J. Wieliczko, S. M. Pryor, M. K. Broadhurst, T. T. Wheeler, and B. J. Haigh. 2011. The abundance of milk cathelicidin proteins during bovine mastitis. Vet. Immunol. Immunopathol. 143:125-130. https://doi.org/10.1016/j.vetimm.2011.06.034.

van Smeden, M., C. A. Naaktgeboren, J. B. Reitsma, K. G. Moons, and J. A. de Groot. 2014. Latent class models in diagnostic studies when there is no reference standard - A systematic review. Am. J. Epidemiol. 179:423-431. https://doi.org/10.1093/aje/kwt286.

Viguier, C., S. Arora, N. Gilmartin, K. Welbeck, and R. O'Kennedy. 2009. Mastitis detection: Current trends and future perspectives. Trends Biotechnol. 27:486-493. https://doi.org/10.1016/j. tibtech.2009.05.004.

Wheeler, T. T., G. A. Smolenski, D. P. Harris, S. K. Gupta, B. J. Haigh, M. K. Broadhurst, A. J. Molenaar, and K. Stelwagen. 2012. Host-defence-related proteins in cows' milk. Animal 6:415-422. https://doi.org/10.1017/S1751731111002151.

Yeaman, M. R., and N. Y. Yount. 2003. Mechanisms of antimicrobial peptide action and resistance. Pharmacol. Rev. 55:27-55. https:// doi.org/10.1124/pr.55.1.2.

Zanetti, M. 2004. Cathelicidins, multifunctional peptides of the innate immunity. J. Leukoc. Biol. 75:39-48. https://doi.org/10.1189/ jlb.0403147.

Zanetti, M. 2005. The role of cathelicidins in the innate host defenses of mammals. Curr. Issues Mol. Biol. 7:179-196. 